\title{
Graphite in 90 T: Evidence for Strong-Coupling Excitonic Pairing
}

\author{
Zengwei Zhu, ${ }^{1,2, *}$ Pan Nie, ${ }^{1}$ Benoît Fauqué, ${ }^{3}$ Baptiste Vignolle, ${ }^{4}$ Cyril Proust, ${ }^{4}$ \\ Ross D. McDonald, ${ }^{2}$ Neil Harrison, ${ }^{2}$ and Kamran Behnia, \\ ${ }^{1}$ Wuhan National High Magnetic Field Center and School of Physics, Huazhong University of Science and \\ Technology, Wuhan 430074, China \\ ${ }^{2}$ MS-E536, NHMFL, Los Alamos National Laboratory, Los Alamos, New Mexico, 87545, USA \\ ${ }^{3}$ JEIP, USR 3573 CNRS, Collège de France, PSL Research University, \\ 11, place Marcelin Berthelot, 75231 Paris Cedex 05, France \\ ${ }^{4}$ Laboratoire National des Champs Magnétiques Intenses (LNCMI-EMFL), CNRS, UGA, UPS, INSA, \\ Grenoble/Toulouse, France \\ ${ }^{5}$ Laboratoire de Physique Et Etude des Matériaux (UPMC-CNRS), ESPCI Paris, PSL Research University \\ 75005 Paris, France
}

(Received 1 November 2018; revised manuscript received 11 January 2019; published 29 March 2019)

Strong magnetic field induces at least two phase transitions in graphite beyond the quantum limit where many-body effects are expected. We report on a study using a state-of-the-art nondestructive magnet allowing us to attain $90.5 \mathrm{~T}$ at $1.4 \mathrm{~K}$, which reveals a new field-induced phase and evidence that the insulating state destroyed at $75 \mathrm{~T}$ is an excitonic condensate of electron-hole pairs. By monitoring the angle dependence of in-plane and out-of-plane magnetoresistance, we distinguish between the role of cyclotron and Zeeman energies in driving various phase transitions. We find that, with the notable exception of the transition field separating the two insulating states, the threshold magnetic field for all other transitions displays an exact cosine angular dependence. Remarkably, the threshold field for the destruction of the second insulator (phase B) is temperature independent with no detectable Landau-level crossing nearby. We conclude that the field-induced insulator starts as a weak-coupling density wave but ends as a strongcoupling excitonic insulator of spin-polarized electron-hole pairs.

DOI: 10.1103/PhysRevX.9.011058

Subject Areas: Condensed Matter Physics

The quantizing effect of high magnetic fields upon the motion of electrons perpendicular to the field orientation effectively reduces the electronic dimensionality of metals. Ultimately, a sufficiently strong magnetic field confines electrons to their lowest Landau level, dramatically increasing the electronic degeneracy at the Fermi energy. In a 2D system, the fractional quantum Hall effect [1] emerges in this regime, due to electron-electron interactions. In 3D systems, on the other hand, electrons can still move freely parallel to the applied field. The quasi-one-dimensionality of their energy spectrum leaves them vulnerable to various instabilities like charge-density wave (CDW), spin-density wave (SDW), excitonic insulator (EI), and valley-density wave $[2,3]$. As a result, the behavior of the threedimensional electron gas in this regime has attracted tremendous attention both experimentally and theoretically

\footnotetext{
*zengwei.zhu@hust.edu.cn
}

Published by the American Physical Society under the terms of the Creative Commons Attribution 4.0 International license. Further distribution of this work must maintain attribution to the author(s) and the published article's title, journal citation, and DOI.
[2-6], albeit with only a limited number of experimental systems with sufficiently low carrier density to reach this limit.

In graphite, beyond its quantum limit (QL) induced by a moderate field applied along the $c$ axis, several phase transitions have been observed [7,8]. In TaP [9] and TaAs [10], the dominant anomalies in resistivity have been attributed to the field-induced annihilation of Weyl nodes reminiscent of the total evacuation of Dirac valleys in bismuth [11]. There are recent reports on the observation of an excitonic insulator in a topological insulator InAs/ GaSb bilayers [12,13] and a semimetal $\mathrm{Ta}_{2} \mathrm{NiSe}_{5}$ [14]. However, only in graphite there is compelling thermodynamic evidence [15] associated with field-induced activation gaps [16], which point to collective electronic phenomena beyond the single-particle picture.

The first experimental observation of an abrupt increase in magnetoresistance around $25 \mathrm{~T}$ at low temperature was reported in 1981 [17]. The domelike phase diagram in the temperature-field plane was established by the study of inplane magnetoresistance $(I \perp c)$. Around $54 \mathrm{~T}$, the insulating state is destroyed [7]. There are several proposals for the identity of the field-induced order, ranging from CDW [18-20], SDW [21,22], EI [23,24], and spin nematic 
excitonic insulator [25]. A more detailed introduction on the theoretical background of these orders can be found in Ref. [8]. Recently, a new transition and a second dome in the temperature-field plane ending at $75 \mathrm{~T}$ were established by out-of-plane $(I \| c)$ measurements [16]. Signatures of these transitions in ultrasonic attenuation and velocity [15] (up to $65 \mathrm{~T}$ ) and in Nernst coefficient [26] in fields below $45 \mathrm{~T}$ have been detected. Exfoliation was also used to study the thickness dependence of the transitions [27].

Here, we present a study of magnetoresistance in a nondestructive magnetic field up to $90.5 \mathrm{~T}$, revealing an additional phase and bringing new insight to the identity of the previously known field-induced states. The contrasting roles of orbital and Zeeman energies can be elucidated by the orientation of the applied field owing to their differing anisotropies. The $\alpha$ and $\beta$ transitions (marking the beginning of the in-plane and out-of-plane insulating response) do not deviate from a cosinusoidal angular dependence over the entire measurement range. In contrast to all other threshold fields, the $54 \mathrm{~T}$ transition $\left(\alpha^{\prime}\right)$ does not follow a cosinusoidal behavior. This implies that the Zeeman energy plays a central role in the transition from one field-induced insulator to the other one. Phase A is located between the $\alpha$ and $\alpha^{\prime}$ anomalies and phase $\mathrm{B}$ between the $\alpha^{\prime}$ and $\zeta$ anomalies. The combination of temperature dependence and angle dependence of the $\zeta$ transition leads us to identify phase $\mathrm{B}$ as a spin-polarized strong-coupling excitonic insulator (likely paring between electron and hole bands with the opposite spin) destroyed by strong magnetic field. By measuring up to $90.5 \mathrm{~T}, \rho_{x x}$ and $\rho_{z z}$, we identify here for the first time a metallic state, beyond the $\zeta$ anomaly, irrespective of the orientation of the charge flow (parallel and perpendicular) respective to the graphene planes. This highlights the peculiarity of phase $\mathrm{B}$, where in-plane metallicity (with no magnetoresistance) coexists with out-of-plane activation. Furthermore, the absence of semimetal to full semiconductor gap at the $\zeta$ transition indicates that in phase $\mathrm{C}$ the lowest spin-polarized hole and electron levels are still occupied.

In-plane magnetoresistance was measured with a standard four-contact setup. Out-of-plane magnetoresistance was measured using two pairs of electrodes attached to the top and bottom of a sample, as illustrated in the inset of Fig. 1. In both cases, the magnetic field was predominantly oriented along the $c$ axis of the sample, with $\theta$ defined to be the angle between the field orientation and the $c$ axis. For the description of samples and measurement methods, see Ref. [28]. Figure 1(a) shows the field dependence of inplane $\rho_{x x}$ and out-of-plane $\rho_{z z}$ magnetoresistance, up to $90.5 \mathrm{~T}$ at $1.4 \mathrm{~K} . \rho_{z z}$ rises by 1 order of magnitude at the $\beta$ transition. It drops before rising again in the vicinity of $54 \mathrm{~T}$, signaling the existence of a second insulating phase named B [16]. It drops again at $75 \mathrm{~T}$ and becomes relatively flat afterward upon the destruction of phase $B$. The in-plane resistivity $\rho_{x x}$ presents a kink at the $\alpha$ transition and a small

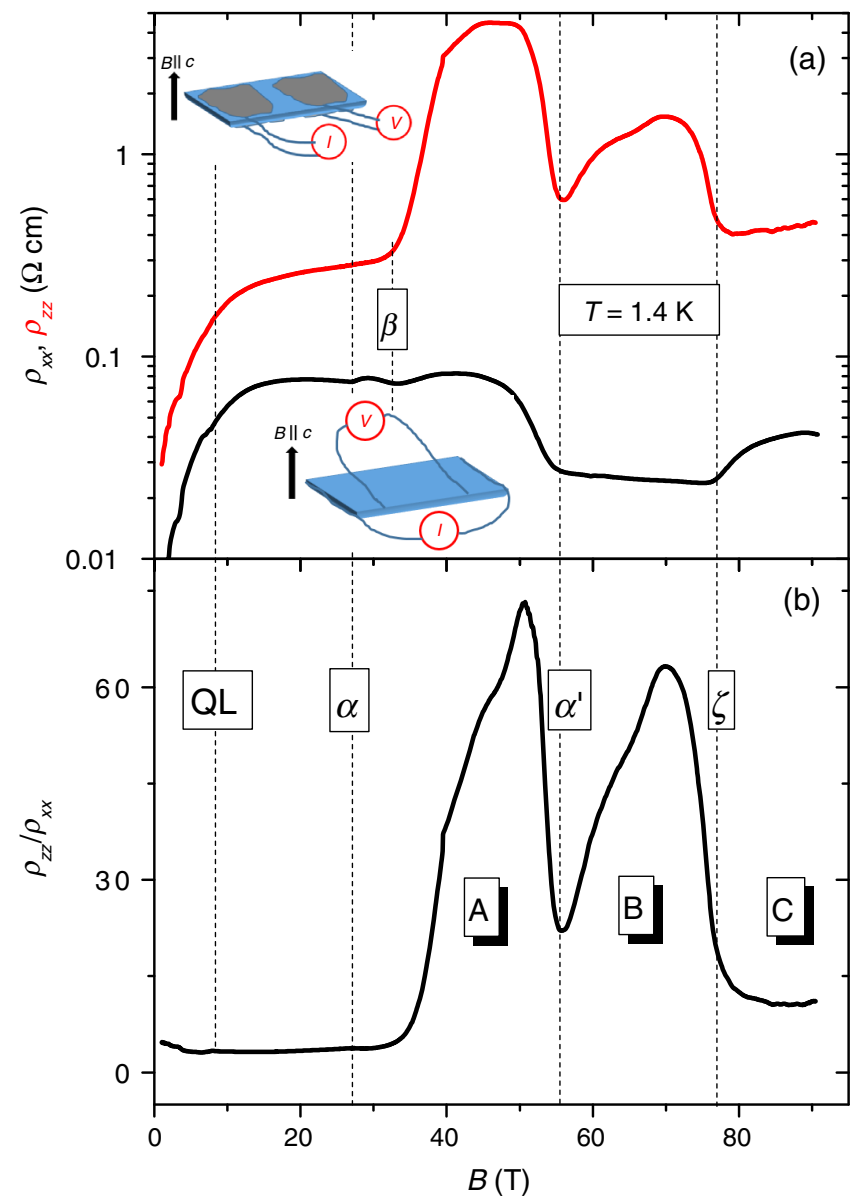

FIG. 1. (a) Field dependence of in-plane magnetoresistance $\rho_{x x}$ and out-of-plane magnetoresistance $\rho_{z z}$ up to $90.5 \mathrm{~T}$ at $1.4 \mathrm{~K}$. The sketches illustrate configurations. (b) The field dependence of ratio $\rho_{z z} / \rho_{x x}$. The quantum limit (QL) and the three phases $(\mathrm{A}, \mathrm{B}$, and $\mathrm{C}$ ) are designated. The zero-field anisotropy is as large as 500. However, it cannot be resolved in our pulsed field data, which is not accurate at low fields.

enhancement at the $\beta$ transition, becomes flat, and then shows a dramatic rise above 75 T. Figure 1(b) displays the field dependence of the anisotropy ratio $\rho_{z z} / \rho_{x x}$. The three field-induced cascading phases can be clearly delineated: phase A refers to the one between the $\alpha$ and $\alpha^{\prime}$ anomalies, phase B exists between the $\alpha^{\prime}$ and $\zeta$ anomalies, and phase $\mathrm{C}$ starts at $\zeta$ and continues up to the highest explored magnetic field. The zero-field anisotropy ratio of the resistivity, $\rho_{z z} / \rho_{x x}$, of the natural graphite sample studied here was found to be around 500, comparable to previous reports $[28,30]$. Upon the application of the magnetic field, $\rho_{z z} / \rho_{x x}$ steadily decreases to around 3 at $7.5 \mathrm{~T}$, above which the quantum limit is reached. Then it increases steeply following the $\beta$ and $\alpha^{\prime}$ transition, recovering significant anisotropy of about 50, still 1 order of magnitude lower than the zero-field value. Finally, following the $\zeta$ transition, the system tends to become quasi-threedimensional again. We note that different types of 

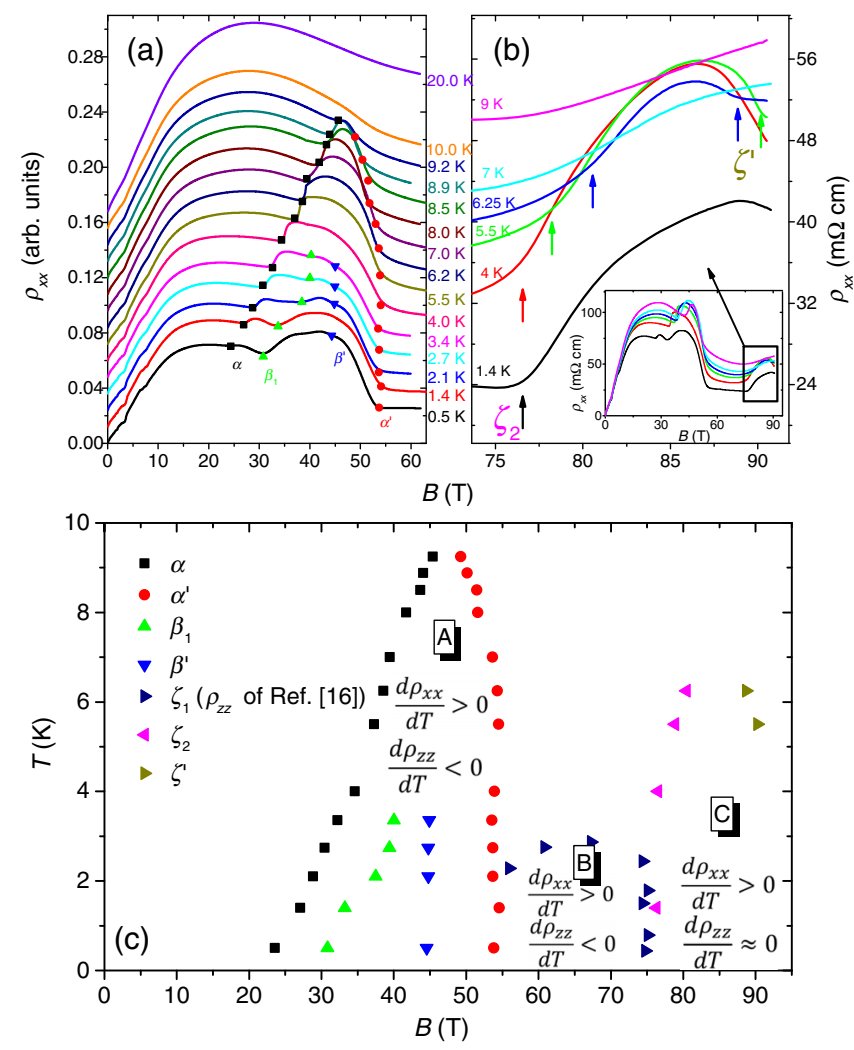

FIG. 2. (a) The field dependence of $\rho_{x x}$ at various temperatures up to $65 \mathrm{~T}$. Curves are shifted for clarity. The different transitions are also labeled accordingly. (b) In-plane magnetoresistance $\rho_{x x}$ (not shifted) between 73 and $90.5 \mathrm{~T}$, while the inset shows magnetoresistance in whole field range at various temperatures. The arrows shows the transitions labeled as $\zeta$ and $\zeta^{\prime}$. (c). The complete phase diagram obtained from the current work. A new phase $\mathrm{C}$ emerges above $75 \mathrm{~T}$.

graphite present different anisotropy ratios [16], and even samples of the same type can be different in their zerofield anisotropies [28]. In spite of these differences, however, the evolution of anisotropy with magnetic field was found to be reproducible [28].

To map out the complete phase diagram, in addition to measuring $\rho_{x x}$ up to $65 \mathrm{~T}$ with a regular pulsed magnet [Fig. 2(a)], we measured $\rho_{x x}$ up to $90.5 \mathrm{~T}$ at different temperatures [Fig. 2(b)]. In Fig. 2(a), curves are shifted for clarity and transitions are identified. Arrows indicate the two new transitions $\zeta_{2}$ and $\zeta^{\prime}$ by our measurements, which encircle a new phase named phase C. Symbols representing $\zeta_{1}$ are taken from the previous $\rho_{z z}$ data [16]. This leads to the field-temperature phase diagram of Fig. 2(c). Phases A and $\mathrm{B}$ were known previously $[15,16]$. In all three phases, the temperature dependence of $\rho_{x x}$ remains metallic. In phase A, $\rho_{z z}$ is insulatinglike while $\rho_{x x}$ is metallic [28]; in phase $\mathrm{B}, \rho_{z z}$ is also insulatinglike while $\rho_{x x}$ is metallic, but the activation gap is smaller [16]. In the new phase $\mathrm{C}, \rho_{z z}$ is almost constant [16] while $\rho_{x x}$ is metallic. Interestingly, the activation gap in the B phase is smaller than that in the

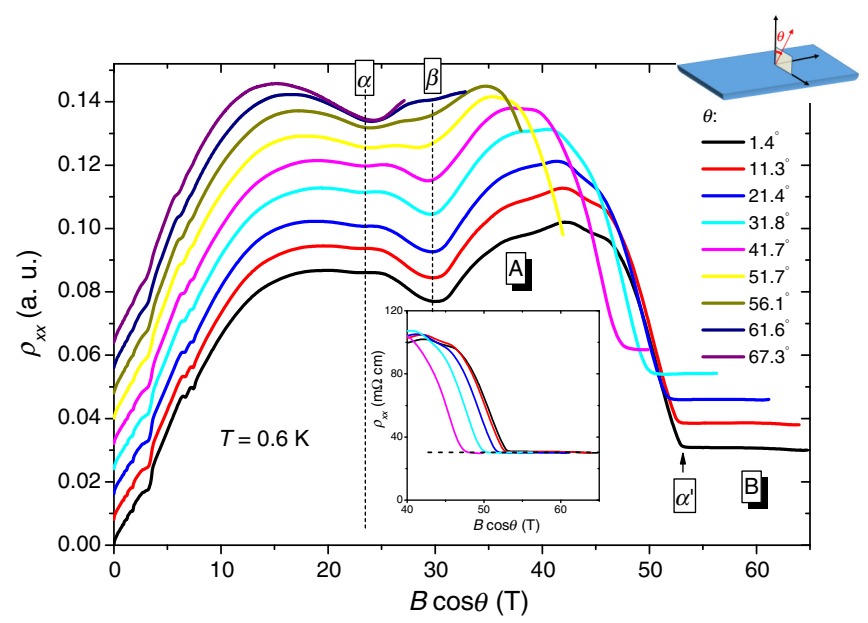

FIG. 3. In-plane magnetoresistance at different angles as a function of $c$-axis component of the magnetic field at $0.6 \mathrm{~K}$. Note that $\alpha$ and $\beta$ remain unchanged, while $\alpha^{\prime}$ changes. Curves are shifted for clarity. The inset shows the same curves with no shift in a limited field window. Note that the flat in-plane resistivity above $\alpha^{\prime}$ remains unchanged.

A phase, in contrast to the upper temperature bound of phase $\mathrm{C}$ being greater than phase $\mathrm{B}$.

In phase $\mathrm{B}$, in-plane resistivity is flat as a function of magnetic field at $0.6 \mathrm{~K}$. As seen in the inset of Fig. 3, when plotted as a function of $B \cos \theta, \rho_{x x}$ curves obtained at different angles collapse on top of each other. This remarkable behavior may be a signature of edge transport in this bulk solid in the quantum limit [31,32]. Assuming that in-plane edge transport in phase B arises because of its insulating nature would provide an explanation for the sudden increase of $\rho_{x x}$ above $75 \mathrm{~T}$. Finite magnetoresistance appears in phase $\mathrm{C}$ because the activation gap (which decoupled layers in phase $\mathrm{B}$ ) is closed in phase $\mathrm{C}$.

Figure 4 shows the magnitude of in-plane conductivity at $64 \mathrm{~T}$ in six different samples. We find that the residual conductance per graphene layer is of the order of $0.03-0.12 e^{2} / h$ in samples in which one can exclude the uncontrolled presence of a component coming from out-ofplane conductivity [28]. This is an order of magnitude lower than minimum conductivity of zero-field graphene [33]. We note that in bulk KHgSb [34], in which the Hall resistance becomes vanishingly small as in our case, a longitudinal conductivity of a similar magnitude has been reported. On the other hand, in bilayer graphene near the neutrality point, edge transport gives rise to a finite longitudinal conductivity of $4 e^{2} / h$ [35]. If the residual in-plane metallicity in our samples is due to edge transport, the elementary $2 \mathrm{D}$ brick should be much thicker than two layers. Theoretically, beyond a thickness of 20 layers, the even-odd dichotomy of layers ends and multilayer graphene becomes graphite [36]. Thus, while attributing the residual in-plane conductivity to a boundary metallicity at the edges of the sample is a tempting scenario, there is 


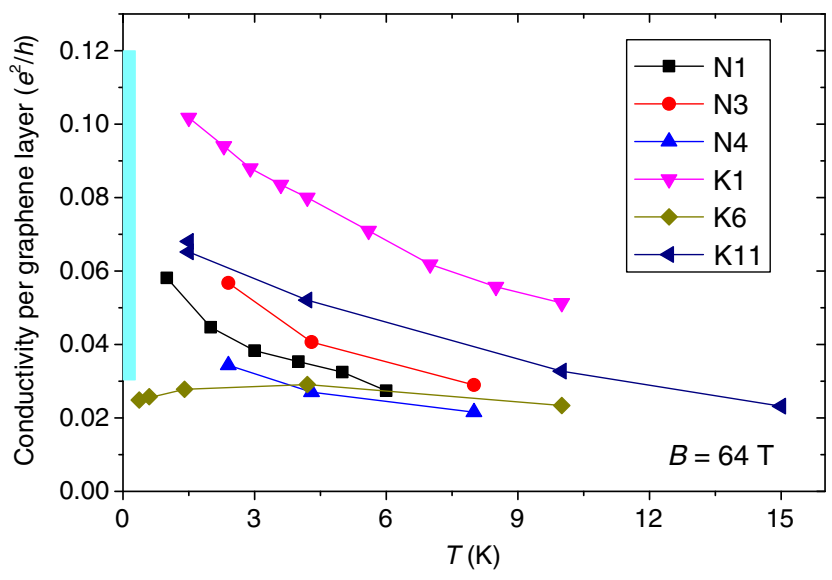

FIG. 4. Temperature dependence of the in-plane conductance per graphene layers in units of $e^{2} / h$ for natural and kish graphite (respectively labeled $\mathrm{N}$ and $\mathrm{K}$ ) at $B=64 \mathrm{~T}$. The conductance per graphene layer is estimated to be $c / 2 t R_{x x}(B=64 \mathrm{~T})$, where $c$ is the $c$-axis length of the hexagonal graphite lattice, $t$ the thickness samples, and $R_{x x}(64 \mathrm{~T})$ the in-plane resistance at $B=64 \mathrm{~T}$. See Ref. [28] for raw data and sample details. The light cyan bar is a guide to the eye representing the uncertainty on the magnitude of the residual conductance per graphene layer.

presently no evidence for any quantization of this transport. Clearly, explaining the origin and the magnitude of in-plane metallicity in phase B needs additional theoretical and experimental efforts.

In graphite spin-orbit coupling is weak and band masses are light; consequently, the Zeeman energy $\frac{1}{2} g \mu_{B} B$ is small compared to the cyclotron energy $\hbar \omega_{c}$ [37]. This shows up in the angle-dependent Nernst data measured at $0.6 \mathrm{~K}$ and up to $17 \mathrm{~T}$ (see Fig. S1 in the Supplemental Material [28]). In contrast to the case of bismuth [38], the angle dependence of the Nernst peaks corresponding to the evacuation of Landau levels is smooth and close to a cosine. The Fermi surface of graphite, composed of two very anisotropic ellipsoids, has a large mass anisotropy (1:7 for the electron ellipsoid, and 1:9 for the hole ellipsoid [39]). Because of this large anisotropy, and the smallness of the Zeeman splitting, the angular dependence of the frequency of quantum oscillations up to $60 \mathrm{deg}$ is close to a cosine fit [39].

In this context, the combination of angle-dependent $\rho_{z z}$ data at $1.4 \mathrm{~K}$ up to $90.5 \mathrm{~T}$ [24] and the angle-dependent Nernst data up to $17 \mathrm{~T}$ reveals a number of important features regarding the role of Zeeman and cyclotron energies in the field-induced cascade. Figure 5 shows how the onset fields for different transitions evolve as the field is tilted off the $c$ axis. As seen in the Fig. 5, except for the $\alpha^{\prime}$ transition, all the others follow a perfect cosine behavior (see Fig. 3 and Supplemental Material [28] for raw data). Interestingly, in Fig. 5(b), the ratio of Zeeman to orbital energies represented from the low field Landaulevel spectrum by $\left(B_{\text {up }}-B_{\text {down }}\right) /\left(B_{\text {up }}+B_{\text {down }}\right)$ presents an angle dependence much weaker than cosinusiodal and strikingly similar to the $\alpha^{\prime}$ transition at $54 \mathrm{~T}$ for $\theta=0$. This indicates that the driving force behind the $\alpha^{\prime}$ transition, which separates two field-induced insulating states, is the Zeeman energy.

By combining the angle dependence and the temperature dependence of the threshold fields, we attempt to address three questions regarding the two phases. (i) How do electrons and holes, which pair up, relate to each other? Are they the occupied and unoccupied states of same Landau subband (the DW scenario) or occupied states of electron and hole subbands (as in the EI case)? (ii) Is the electron-hole condensate in the weak-coupling or in the strong-coupling limit? (iii) Do pairing electrons and holes have parallel or opposite spins? Above the QL at 7.5 T, only four subbands (electrons and holes, each with up and down

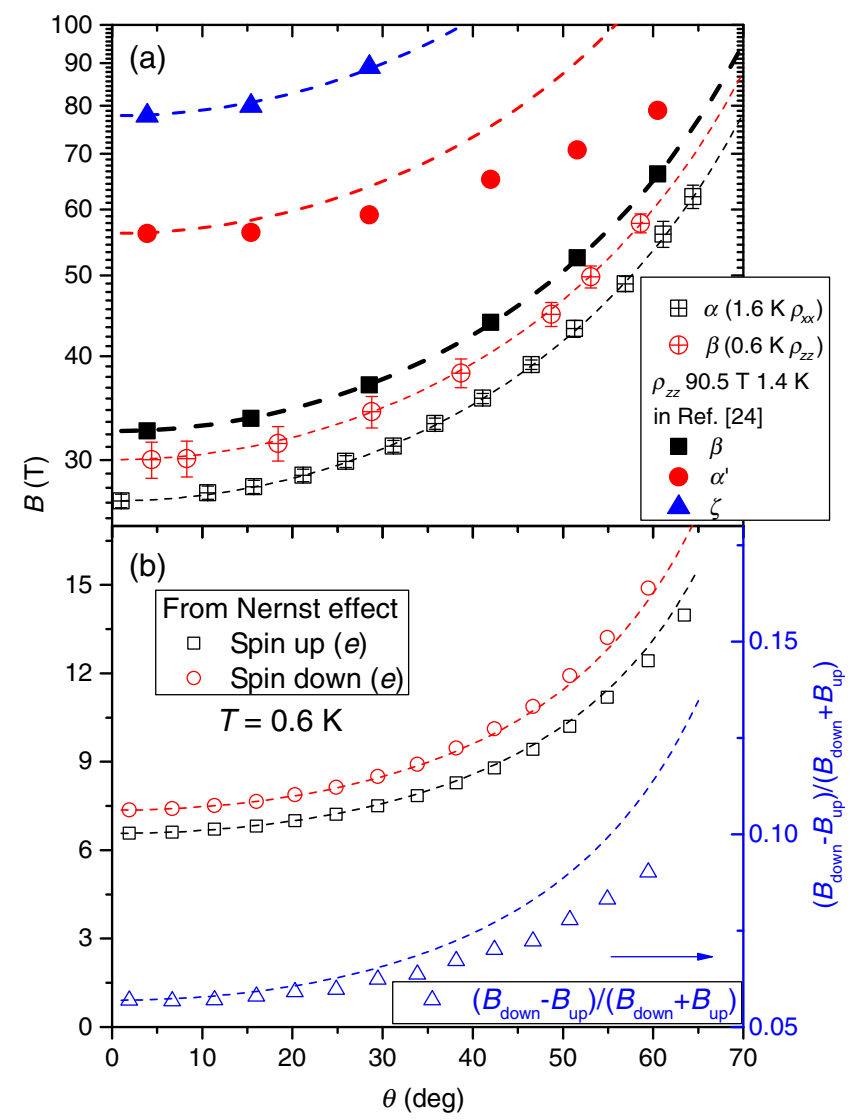

FIG. 5. (a) The angle dependence of the threshold fields. Dashed lines represent a cosine dependence. Except for $B_{\alpha^{\prime}}$, all other transitions display a cosine behavior. The " + " center symbols represent anomalies in magnetoresistance data up to $65 \mathrm{~T}$ with regular pulse magnets [28]. The solid symbols represent anomalies in $\rho_{z z}$ up to $90.5 \mathrm{~T}$ [24]. (b) The angle dependence of the Nernst peaks identified with the evacuation of spin-up and spin-down $(n=1)$ subbands. Also shown is the angle dependence of the $\left(B_{\text {up }}-B_{\text {down }}\right) /\left(B_{\text {up }}+B_{\text {down }}\right)$ ratio. Note the similarity between the behavior of this ratio which quantifies the ratio of Zeeman-toorbital energy and the behavior of the transition $\alpha^{\prime}$ in (a). 
spins) remain occupied. Figure 6 shows a sketch of the Landau-level spectrum above the QL when four or two Landau subbands are occupied. Phase A, relatively easy to access by regular pulsed magnetic field, was intensely studied before. Its threshold magnetic field $(\alpha)$ is strongly temperature dependent and its angular dependence follows a cosine dependence. The temperature dependence can be described by a weak-coupling BCS formula $[7,8,18]$, where the density of states (DOS) is set by the Landau-level degeneracy. Its perfect cosine field dependence [Fig. 5(b)] is to be contrasted by the angle dependence of a Landau level, like the one evacuated at $7.5 \mathrm{~T}$ [Fig. 5(b)]. The comparison suggests that the Zeeman energy does not play any role in driving the $\alpha$ transition. This would be true no matter the mutual orientation of spins of electrons and holes. Even holes and electrons with opposite spins, the paired quasiparticle can cancel out their Zeeman energy, leaving the orbital term with an exactly cosine angular dependence. The same can be said about the $\beta$ phase

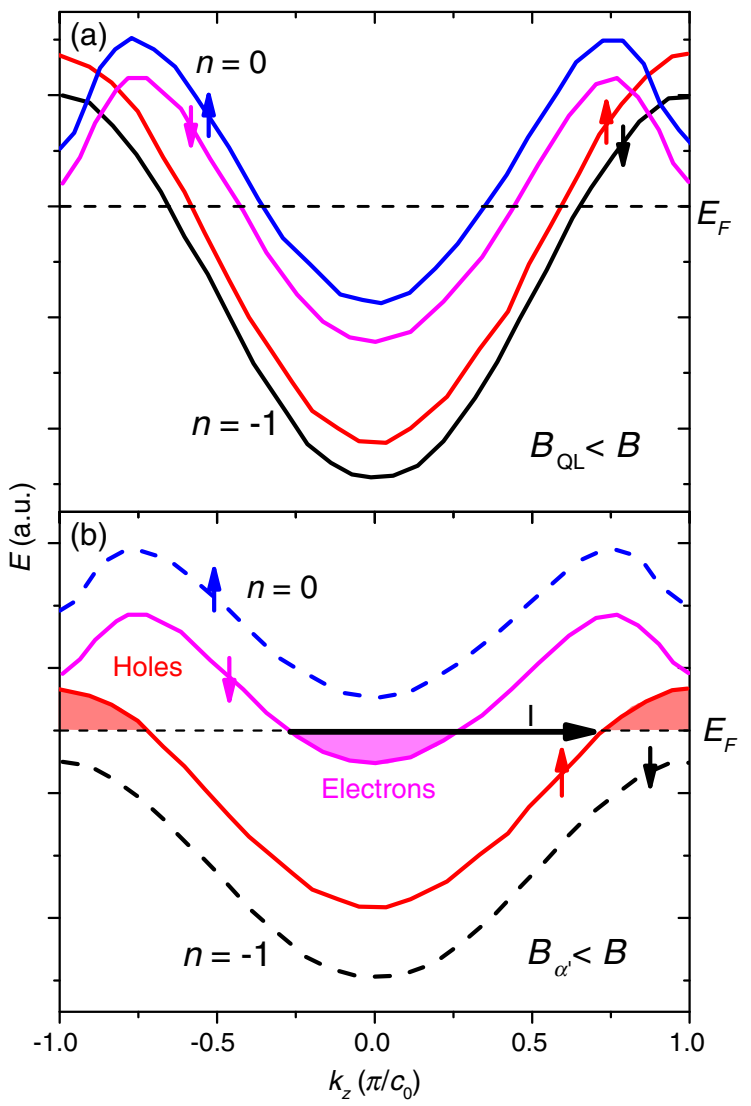

FIG. 6. (a) and (b) Sketch of the Landau-level spectrum of graphite below and above $52 \mathrm{~T}$ respectively according to the current theoretical works. Between 7.5 and $52 \mathrm{~T}$, four Landau levels are occupied: two for the electrons pockets $(0, \uparrow),(0, \downarrow)$ and two for the hole pockets $(-1, \uparrow),(-1, \downarrow)$. Above $52 \mathrm{~T}$, only the $(0, \downarrow)$ and $(-1, \uparrow)$ are occupied. We colored in purple and red the occupied states in the lowest electron and hole Laundau levels in (b). The vector I denotes the possible intersubband nesting vector for the excitonic phase. transition. Phase $\mathrm{B}$ was discovered using an $80 \mathrm{~T}$ magnet [16]. The $\alpha^{\prime}$ transition separating $\mathrm{A}$ and $\mathrm{B}$ phases has almost no temperature dependence. It is accompanied by the depopulation of at least one and most probably two Landau levels $[7,15,19,23,24]$. According to the present study, the angle dependence of this $\alpha^{\prime}$ transition, which separates two insulators with different gaps [16], points to Zeeman energy as its driving source. One possibility is that the nature of the condensate is different in the two phases (see Supplemental Material [28] for a more detailed discussion). The $\zeta$ transition field separating phase B and $\mathrm{C}$ has almost no temperature dependence and its angle dependence follows a cosine behavior. Up to $90.5 \mathrm{~T}$, both $\rho_{x x}$ and $\rho_{z z}$ remain metallic and the Hall coefficient is null, consistent with Ref. [24]. As a consequence, one can rule out the evacuation of the two remaining Landau sublevels and the subsequent semimetal to semiconductor transition previously postulated [19]. Therefore the field-induced destruction of phase $\mathrm{C}$ cannot be attributed to any change in the density of states in the vicinity of a Landau-level evacuation. If the electrons and holes which pair up belong to different subbands, their different Fermi velocities would provide an opportunity for a strong magnetic field to unbind them through a purely orbital effect. An alternative scenario invoking spin quantum fluctuations destabilizing the EI was recently proposed by Pan et al. [25]. Neither of these scenarios for the field-induced destruction of phase $\mathrm{C}$ would work in the case of a density wave.

Like a DW, EI is a condensate of electron-hole pairs, but unlike it, electrons and holes which pair up belong to different subbands located at different points of the reciprocal space at zero field and different dispersions along the field orientation in the quantum limit. In Fig. 6(b), electron and hole pockets in the B phase are shown in purple and red. The vector driving electron-hole pairing in the EI scenario is labeled I. It couples two points of the lowest electron and hole Landau levels. Observations reported here all converge in favoring the EI scenario at least in the very strong field limit (above $50 \mathrm{~T}$ ).

In summary, using a very strong magnetic field, we found an additional phase transition above $75 \mathrm{~T}$ and dubbed it phase C. By monitoring the angle dependence of the multiple fields inducing phase transitions, we found that the threshold fields for all transitions, save one $\left(\alpha^{\prime}\right)$, show exact cosine dependence in angle. The in-plane magnetoresistance in phase B indicates edge transport. There is no insulating state even at $90 \mathrm{~T}$ and the field-induced destruction of phase $\mathrm{B}$ is not accompanied by the evacuation of any Landau levels. The combination of these facts leads us to conclude that phase $\mathrm{B}$ is an excitonic insulator, a spinpolarized condensate of electron-hole pairs.

\section{ACKNOWLEDGMENTS}

We acknowledge useful discussions with Ryuichi Shindou. This work in China was supported by the 
National Science Foundation of China (Grants No. 11574097 and No. 1861135104), the National Key Research and Development Program of China (Grant No. 2016YFA0401704). Z. Z. was also supported by the 1000 Youth Talents Plan in China and the directors funding Grant No. 20120772 at LANL. N.H. and R. D. M. acknowledge support from the U.S. DOE BES "Science of 100T" program. The National High Magnetic Field Laboratory-PFF facility is funded by the National Science Foundation Cooperative Agreements No. DMR-1157490 and No. DMR-1644779, the State of Florida, and the U.S. Department of Energy. In France, this work was part of QUANTUM LIMIT projects funded by Agence Nationale de la Recherche. B. F. acknowledges support from Jeunes Equipes de 1'Institut de Physique du Collège de France (JEIP). K. B. was supported by China High-end foreign expert program, 111 Program, and Fonds-ESPCI-Paris. We acknowledge support from the LNCMI, a member of the European Magnetic Field Laboratory (EMFL). We acknowledge the supports from our engineering teams in the three pulsed field facilities.

[1] H. L. Stormer, D. C. Tsui, and A. C. Gossard, The Fractional Quantum Hall Effect, Rev. Mod. Phys. 71, S298 (1999).

[2] B. I. Halperin, Possible States for a Three-Dimensional Electron Gas in a Strong Magnetic Field, Jpn. J. Appl. Phys. 26, 1913 (1987).

[3] A. H. MacDonald and G. W. Bryant, Strong-Magnetic-Field States of the Pure Electron Plasma, Phys. Rev. Lett. 58, 515 (1987).

[4] V. Celli and N. D. Mermin, Ground State of an Electron Gas in a Magnetic Field, Phys. Rev. 140, A839 (1965).

[5] H. J. Lee, Density Fluctuations and Ground State for a System of Electrons in a Strong Magnetic Field, Phys. Rev. 177, 786 (1969).

[6] Z. Tesanović and B. I. Halperin, Multivalley Electron Gas in a Strong Magnetic Field, Phys. Rev. B 36. 4888 (1987).

[7] H. Yaguchi and J. Singleton, A High-Magnetic-FieldInduced Density-Wave State in Graphite, J. Phys. Condens. Matter 21, 344207 (2009).

[8] B. Fauqué and K. Behnia, in Basic Physics of Functionalized Graphite, edited by P.D. Esquinazi (Springer, New York, 2016), Chap. 4.

[9] C. L. Zhang et al., Magnetic-Tunnelling-Induced Weyl Node Annihilation in TaP, Nat. Phys. 13, 979 (2017).

[10] B. J. Ramshaw et al., Quantum Limit Transport and Destruction of the Weyl Nodes in TaAs, Nat. Commun. 9, 2217 (2018).

[11] Z. Zhu, J. Wang, H. Zuo, B. Fauqué, R. D. McDonald, Y. Fuseya, and K. Behnia, Emptying Dirac Valleys in Bismuth Using High Magnetic Fields, Nat. Commun. 8, 15297 (2017).

[12] L. Du, X. Li, W. Lou, G. Sullivan, K. Chang, J. Kono, and R.-R. Du, Evidence for a Topological Excitonic Insulator in InAs/GaSb Bilayers, Nat. Commun. 8, 1971 (2017).
[13] W. Yu et al., Anomalously Large Resistance at the Charge Neutrality Point in a Zero-Gap InAs/GaSb Bilayer, New J. Phys. 20, 053062 (2018).

[14] Y. F. Lu, H. Kono, T. I. Larkin, A. W. Rost, T. Takayama, A. V. Boris, B. Keimer, and H. Takagi, Zero-Gap Semiconductor to Excitonic Insulator Transition in $\mathrm{Ta}_{2} \mathrm{NiSe}_{5}$, Nat. Commun. 8, 14408 (2017).

[15] D. LeBoeuf, C. W. Rischau, G. Seyfarth, R. Küchler, M. Berben, S. Wiedmann, W. Tabis, M. Frachet, K. Behnia, and B. Fauqué, Thermodynamic Signatures of the Field-Induced States of Graphite, Nat. Commun. 8, 1337 (2017).

[16] B. Fauqué, D. LeBoeuf, B. Vignolle, M. Nardone, C. Proust, and K. Behnia, Two Phase Transitions Induced by a Magnetic Field in Graphite, Phys. Rev. Lett. 110, 266601 (2013).

[17] S. Tanuma, R. Inada, A. Furukawa, O. Takahashi, Y. Iye, and Y. Onuki, in Physics in High Magnetic Fields, edited by S. Chikazumi and N. Miura,Springer Series in SolidState Sciences Vol. 24 (Springer, Berlin, Heidelberg, 1981), p. 316.

[18] D. Yoshioka and H. Fukuyama, Electronic Phase Transition of Graphite in a Strong Magnetic Field, J. Phys. Soc. Jpn. 50, 725 (1981).

[19] F. Arnold, A. Isidori, E. Kampert, B. Yager, M. Eschrig, and J. Saunders, Charge Density Waves in Graphite; Towards the Magnetic Ultraquantum Limit, Phys. Rev. Lett. 119, 136601 (2017).

[20] X.-T. Zhang and R. Shindou, Transport Properties of Density Wave Phases in Three-Dimensional Metals and Semimetals under High Magnetic Field, Phys. Rev. B 95, 205108 (2017).

[21] Y. Takada and H. Goto, Exchange and Correlation Effects in the Three-Dimensional Electron Gas in Strong Magnetic Fields and Application to Graphite, J. Phys. Condens. Matter. 10, 11315 (1998).

[22] K. Takahashi and Y. Takada, Charge- and Spin-DensityWave Instabilities in High Magnetic Fields in Graphite, Physica (Amsterdam) 201B, 384 (1994).

[23] K. Akiba, A. Miyake, H. Yaguchi, A. Matsuo, K. Kindo, and M. Tokunaga, Possible Excitonic Phase of Graphite in the Quantum Limit, J. Phys. Soc. Jpn. 84, 054709 (2015).

[24] Z. Zhu, R. D. McDonald, A. Shekhter, B. J. Ramshaw, K. A. Modic, F. F. Balakirev, and N. Harrison, Magnetic Field Tuning of an Excitonic Insulator between the Weak and Strong Coupling Regimes in Quantum Limit Graphite, Sci. Rep. 7, 1733 (2017).

[25] Z. Pan, X. T. Zhang, and R. Shindou, Theory of MetalInsulator Transitions in Graphite under High Magnetic Field, Phys. Rev. B 98, 205121 (2018).

[26] B. Fauqué, Z. Zhu, T. Murphy, and K. Behnia, Nernst Response of the Landau Tubes in Graphite across the Quantum Limit, Phys. Rev. Lett. 106, 246405 (2011).

[27] T. Taen, K. Uchida, and T. Osada, Thickness-Dependent Phase Transition in Graphite under High Magnetic Field, Phys. Rev. B 97, 115122 (2018).

[28] See Supplemental Material at http://link.aps.org/ supplemental/10.1103/PhysRevX.9.011058 for sample description, angular dependence transport measurement, another set of data up to $80 \mathrm{~T}$ measured in WHMFC, 
temperature dependence of $\rho_{x x}$ in the phase B and possible pairings for different phases, which includes Ref. [29].

[29] Z. Zhu, H. Yang, B. Fauqué, Y. Kopelevich, and K. Behnia, Nernst Effect and Dimensionality in the Quantum Limit, Nat. Phys. 6, 26 (2010).

[30] W. Primak and L. H. Fuchs, Electrical Conductivities of Natural Graphite Crystals, Phys. Rev. 95, 22 (1954).

[31] B. A. Bernevig, T. L. Hughes, S. Raghu, and D. P. Arovas, Theory of the Three-Dimensional Quantum Hall Effect in Graphite, Phys. Rev. Lett. 99, 146804 (2007).

[32] L. Balents and M. P. A. Fisher, Chiral Surface States in the Bulk Quantum Hall Effect, Phys. Rev. Lett. 76, 2782 (1996).

[33] K. Ziegler, Minimal Conductivity of Graphene: Nonuniversal Values from the Kubo Formula, Phys. Rev. B 75, 233407 (2007).

[34] S. Liang, S. Kushwaha, T. Gao, M. Hirschberger, J. Li, Z. Wang, K. Stolze, B. Skinner, B. A. Bernevig, R. J. Cava, and N. P. Ong, A Gap-Protected Zero-Hall Effect State in the Quantum Limit of the Nonsymmorphic Metal KHgSb, arXiv:1812.11912.
[35] K. S. Novoselov, E. McCann, S. V. Morozov, V. I. Fal'ko, M. I. Katsnelson, U. Zeitler, D. Jiang, F. Schedin, and A. K. Geim, Unconventional Quantum Hall Effect and Berry's Phase of $2 \pi$ in Bilayer Graphene, Nat. Phys. 2, 177 (2006).

[36] B. Partoens and F. M. Peeters, From graphene to graphite: Electronic structure around the $K$ point, Phys. Rev. B 74, 075404 (2006).

[37] Y. Fuseya, Z. Zhu, B. Fauqué, W. Kang, B. Lenoir, and K. Behnia, Origin of the Large Anisotropic g-Factor of Holes in Bismuth, Phys. Rev. Lett. 115, 216401 (2015).

[38] Z. Zhu, B. Fauqué, Y. Fuseya, and K. Behnia, Angleresolved Landau spectrum of electrons and holes in bismuth, Phys. Rev. B 84, 115137 (2011).

[39] J. M. Schneider, B. A. Piot, I. Sheikin, and D. K. Maude, Using the de Haas-van Alphen Effect to Map Out the Closed Three-Dimensional Fermi Surface of Natural Graphite, Phys. Rev. Lett. 108, 117401 (2012). 